\title{
Gödel and the Incompleteness of Arithmetic
}

\author{
Pinheiro \\ IICSE University, Wilmington, USA \\ Email: drmarciapinheiro@gmail.com
}

Received 1 June 2016; accepted 12 July 2016; published 15 July 2016

Copyright (C) 2016 by author and Scientific Research Publishing Inc.

This work is licensed under the Creative Commons Attribution International License (CC BY).

http://creativecommons.org/licenses/by/4.0/

c) (i) Open Access

\begin{abstract}
People normally believe that Arithmetic is not complete because Gödel launched this idea a long time ago, and it looks as if nobody has presented sound evidence on the contrary. We here intend to do that perhaps for the first time in history. We prove that what Stanford Encyclopedia has referred to as Theorem 3 cannot be true, and, therefore, if nothing else is presented in favour of Gödel's thesis, we actually do not have evidence on the incompleteness of Arithmetic: All available evidence seems to point at the extremely opposite direction.
\end{abstract}

\section{Keywords}

Gödel, Arithmetic, Peano, Axiom, Classical Logic

\section{Introduction}

From [1]:

Kurt Gödel was born on April 28, 1906 in what was then the Austro-Hungarian city of Brünn, and what is now Brno in the Czech Republic.

Gödel proved to be an exemplary student at primary school and later the Gymnasium, excelling especially in mathematics, languages and religion. Upon his graduation from the Gymnasium in Brno in 1924, Gödel enrolled in the University of Vienna, attending lectures on physics, his initial field of interest, lectures on philosophy given by Heinrich Gomperz, and lectures on mathematics. Gödel took a number of physics courses during his undergraduate years, as witnessed by his university transcript; this is notable in view of Gödel's subsequent contributions to relativity in 1947. Philipp Furtwängler, cousin of the great German conductor Wilhelm Furtwängler, was one of his mathematics professors, and indeed Furtwängler's course on class field theory almost tempted Gödel to pursue his studies in that area. Gödel learned his logic from Rudolph Carnap and from Hans Hahn, eventually graduating under Hahn with a Dr. Phil. in mathematics in 1929. The main theorem of his dissertation was the completeness theorem for first order logic [2].

After reading the above extract, we get to the understanding that Gödel was mostly a mathematician, is it not? 
About the completeness theorem for First Order Logic [1]:

The completeness question for the first order predicate calculus was stated precisely and in print for the first time in 1928 by Hilbert and Ackermann in their text Grundzüge der theoretischen Logik [3], a text with which Gödel would have been quite familiar. [6]

The question Hilbert and Ackermann pose is whether a certain explicitly given axiom system for the first order predicate calculus "...is complete in the sense that from it all logical formulas that are correct for each domain of individuals can be derived...” ([4], p. 48).

Proof of the Completeness Theorem

We give an outline of Gödel's own proof in his doctoral thesis [2]. An essential difference with earlier efforts (discussed below and elsewhere, e.g. in [5]), is that Gödel defines meticulously all the relevant basic concepts.

A "logical expression" in Gödel's terminology is a well-formed first order formula without identity. An expression is "refutable" if its negation is provable, "valid" if it is true in every interpretation and "satisfiable" if it is true in some interpretation. The Completeness Theorem is stated as follows:

\section{Theorem 1}

Every valid logical expression is provable. Equivalently, every logical expression is either satisfiable or refutable.

Gödel's proof calculus is that of Hilbert and Ackermann's text. An expression is in normal form if all the quantifiers occur at the beginning. The degree of an expression or formula is the number of alternating blocks of quantifiers at the beginning of the formula, assumed to begin with universal quantifiers. Gödel shows that if the completeness theorem holds for formulas of degree $\mathrm{k}$, it must hold for formulas of degree $\mathrm{k}+1$. Thus the question of completeness reduces to formulas of degree 1 . That is, it is to be shown that any normal formula (Q) $\varphi$ of degree 1 is either satisfiable or refutable, where "(Q)" stands for a (non-empty) block of universal quantifiers followed by a (possibly empty) block of existential ones.

Gödel defines a book-keeping device, a well-ordering of all tuples of variables arising from a need to satisfy $\varphi$ as dictated by $(\mathrm{Q})$. For example, if $(\mathrm{Q}) \varphi$ is $\forall \mathrm{x}_{0} \exists \mathrm{x}_{1} \psi\left(\mathrm{x}_{0}, \mathrm{x}_{1}\right)$, we list the quantifier-free formulas $\psi\left(\mathrm{x}_{\mathrm{n}}, \mathrm{x}_{\mathrm{n}+1}\right)$. (Or more precisely, finite conjunctions of these in increasing length. See below.) Then in any domain consisting of the values of the different $\mathrm{x}_{\mathrm{n}}$, in which each $\psi\left(\mathrm{x}_{\mathrm{n}}, \mathrm{x}_{n+1}\right)$ is true, the sentence $(\mathrm{Q}) \varphi$ is clearly true. A crucial lemma claims the provability, for each $\mathrm{k}$, of the formula $(\mathrm{Q}) \varphi \rightarrow\left(\mathrm{Q}_{\mathrm{k}}\right) \varphi_{\mathrm{k}}$, where the quantifier free formula $\varphi_{\mathrm{k}}$ asserts the truth of $\psi$ for all tuples up to the kth tuple of variables arising from $(\mathrm{Q})$, and $\left(\mathrm{Q}_{\mathrm{k}}\right) \varphi_{\mathrm{k}}$ is the existential closure of $\varphi_{\mathrm{k}}$. (See the example below where the definition of the $\varphi_{\mathrm{k}}$ s is given.) This lemma is the main step missing from the various earlier attempts at the proof due to Löwenheim and Skolem, and, in the context of the completeness theorem for first order logic, renders the connection between syntax and semantics completely explicit.

Let us consider an example of how a particular formula would be found to be either satisfiable or its negation provable, following Gödel' $s$ method: Consider $\varphi=\forall \mathrm{x}_{0} \exists \mathrm{x}_{1} \psi\left(\mathrm{x}_{0}, \mathrm{x}_{1}\right)$, where $\psi\left(\mathrm{x}_{0}, \mathrm{x}_{1}\right)$ is quantifier-free. We show that this is either refutable or satisfiable. We make the following definitions:

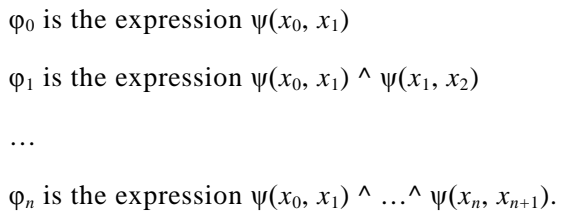

The crucial lemma, referred to above, shows that from $\varphi$ we can derive for each $\mathrm{n}, \exists \mathrm{x}_{0} \ldots \exists \mathrm{x}_{\mathrm{n}+1} \varphi_{\mathrm{n}}$.

Case 1: For some n, $\varphi_{\mathrm{n}}$ is not satisfiable. Then, Gödel argued, using the already known completeness theorem for propositional logic, [7] that $\neg \varphi_{\mathrm{n}}$ is provable, and hence so is $\forall \mathrm{x}_{0}, \ldots, \mathrm{x}_{\mathrm{n}+1} \neg \varphi_{\mathrm{n}}$. Thus $\neg \exists \mathrm{x}_{0} \ldots \exists \mathrm{x}_{\mathrm{n}+1} \varphi_{\mathrm{n}}$ is provable and therefore the $\neg \varphi$ is provable, i.e., $\varphi$ is refutable in the Hilbert-Ackermann system. (Some partial results about propositional logic in addition to those already mentioned include the semantic completeness of the propositional calculus due to [6], as well as a more general completeness theorem for the same due to Bernays in 1918; the latter appears in Bernays' unpublished Habilitationsschrift of 1918; see also [7].)

Case 2: Each $\varphi_{\mathrm{n}}$ is satisfiable. There are only finitely many possible models with universe $\left\{\mathrm{x}_{0}, \ldots, \mathrm{x}_{n+1}\right\}$. Gödel orders them as a tree by defining a model $\mathrm{M}$ to be below a model $\mathrm{M}^{\prime}$ if $\mathrm{M}$ is a submodel of $\mathrm{M}^{\prime}$. In this way we obtain a tree which is finitely branching but infinite. By König's Lemma there is an infinite branch B. (In the proof, 
Gödel explicitly constructs the branch given by König's Lemma rather than citing it by name). The union of the models on $\mathrm{B}$ forms a model $\mathrm{M}$ with universe $\left\{\mathrm{x}_{0}, \mathrm{x}_{1}, \ldots\right\}$. Since $\mathrm{M}$ satisfies each $\varphi_{\mathrm{n}}$, the original formula $\varphi$ holds in M. So $\varphi$ is satisfiable and we are done.

Note that the model, in the satisfiability case of Gödel's proof, is always countable. Thus this proof of the Completeness Theorem gives also the Löweheim-Skolem Theorem (see below). Gödel extends the result to countably many formulas and to the case of first order logic with identity. He also proves the independence of the axioms.

In 1930 Gödel published the paper based on his thesis [8] notable also for the inclusion of the compactness theorem, which is only implicitly stated in the thesis. The theorem as stated by Gödel in [8] is as follows: a countably infinite set of quantificational formulas is satisfiable if and only if every finite subset of those formulas is satisfiable. Gödel uses compactness to derive a generalization of the completeness theorem.

The Compactness Theorem was extended to the case of uncountable vocabularies by Maltsev in 1936 [9], from which the Upward Löwenheim-Skolem theorem immediately follows. The Compactness Theorem would become one of the main tools in the then fledgling subject of model theory.

Please pay attention to the definition of completeness that Gödel here presents because he seems to violate it in what people claim to be his proof of incompleteness: Every valid logical expression is provable.

Also pay attention to his definitions (as for the above extract): A logical expression in Gödel's terminology is a well-formed first order formula without identity. An expression is refutable (false) if its negation is provable, valid if it is true in every interpretation and satisfiable if it is true in some interpretation.

Now we are in trouble!

The same source we mentioned above (Stanford Encyclopedia) states the following:

The First Incompleteness Theorem provides a counterexample to completeness by exhibiting an arithmetic statement which is neither provable nor refutable in Peano arithmetic, though true in the standard model.

Theorem 3 (Gödel's First Incompleteness Theorem)

If $P$ is $\omega$-consistent, then there is a sentence which is neither provable nor refutable from $P$.

Proof: By judicious coding of syntax referred to above, write a formula $\operatorname{Prf}(x, y)$ of number theory, representable in $P$, so that

1. $n$ codes a proof of $\varphi \Rightarrow P \vdash \operatorname{Prf}(n, \digamma \varphi))$.

and

2. $n$ does not code a proof of $\varphi \Rightarrow P \vdash \neg \operatorname{Prf}(n, / \varphi)$.

Let Prov(y) denote the formula $\exists x \operatorname{Prf}(x, y)$. By Theorem 2 there is a sentence $\varphi$ with the property

3. $P \vdash\left(\varphi \leftrightarrow \neg \operatorname{Prov}\left(\digamma_{\varphi}\right)\right)$.

Thus $\varphi$ says 'I am not provable.' We now observe, if $P \vdash \varphi$, then by (1) there is $n$ such that $P \vdash \operatorname{Prf}(n, I \varphi)$, hence $P \vdash \operatorname{Prov}((\varphi))$, hence, by (3) $P \vdash \neg \varphi$, so $P$ is inconsistent. Thus

\section{4. $P \not \nvdash \varphi$}

Furthermore, by (4) and (2), we have $P \vdash \neg \operatorname{Prf}(n, I \varphi)$ for all natural numbers $n$. By $\omega$-consistency $P \Vdash \exists x \operatorname{Prf}(x$, $\ulcorner\varphi)$. Thus (3) gives $P \nvdash \neg \varphi$. We have shown that if $P$ is $\omega$-consistent, then $\varphi$ is independent of $P$.

In this paper, we will attempt to discuss the assertions just presented from a totally unbiased perspective.

Our conclusion should be that no actual proof of incompleteness of Arithmetic was ever presented.

\section{Development}

The source (Stanford Encyclopedia) is correct in stating that this possible evidence on incompleteness has something that is similar to the Liar Paradox, but it is not correct to state that the possible evidence on incompleteness and the Liar Paradox are the same thing.

We observe that the Liar Paradox involves human elements (a person said, believes, human definition of truth, etc.), as one can see on [10].

The just-mentioned fact makes of The Liar something completely different from the evidence Stanford Encyclopedia claims that Gödel had on the incompleteness of Arithmetic.

Gödel coded his proof, so that there is no human element in any of it, which is the ideal [11].

Peano's Arithmetic is defined as follows [12]:

1. Zero is a number.

2. If $a$ is a number, the successor of $a$ is a number.

3. Zero is not the successor of a number. 
4. Two numbers of which the successors are equal are themselves equal.

5. (Induction Axiom) If a set $S$ of numbers contains zero, and also the successor of every number in $S$, then every number is in $S$.

Peano's axioms are the basis for the version of number theory known as Peano Arithmetic.

We observe that there is nothing similar to what Stanford says that Gödel referred to in the above axioms.

The whole thing was about number 3:

$P \vdash(\varphi \leftrightarrow \neg \operatorname{Prov}(\lceil\varphi))$.

We observe the following: Number 3 cannot be true in Classical Logic. Notice that a CL implication will only be untrue if we have $1->0$ [13].

Now the problem arises: With this assertion, we not only have evidence on the possible inconsistency of Arithmetic, but also a piece of evidence on the possible inconsistency of CL, and therefore in Mathematics in general. Observe that when the antecedent, $\varphi$, is true, what then could be expressed as $v(\varphi)=1$, and the consequent is untrue, what then could be expressed as $v(\neg \operatorname{Prov}(I \varphi))=0$, we have, as a consequence, due to the allowed truth-values in CL, that $v(\operatorname{Prov}(I \varphi))=1$. In this case, it is not possible to have $1->0$ in this particular statement, what means that it is always true or that it is a tautology. $P$ should always support any tautology, so that we know that this is true by default. The problem then becomes who is $\varphi$, is it not? We further observe that things go funny also in a $1->1$ situation. In this case, $v(\varphi)=1$ and $v(\neg \operatorname{Prov}(\lceil\varphi\rceil))=1$. The implication should always be true in CL, so that we are obliged to admit that we do not need to have a proof of $\varphi$ to say that $v(\varphi)=1$ in our system, which should be CL. Assume then that, even though we do not need a proof to say that something is true, if we have that something has a proof, that implies that something is true. In this case, we have the unwanted tautology, so that we would have to say that, in such a system, it is not the case that if we have a proof of something, that something is true, we reckon. Otherwise, we will be voiding a major law in CL, which is that $1->0$ implies 0 , not 1 .

We know that everything in Arithmetic should be provable, apart from the axioms.

If we then say that we do not need proofs to say that something is true, we can accept the axioms as part of Arithmetic, we reckon, and perhaps as part of any system.

The problem comes from when we have a proof, since now things could be false even with a proof.

We probably want to preserve this system of proving somehow that $2+3$ is 5 , then calling that true only after the proof is written.

If we do want to preserve that, it is possible that Gödel is right and we then have an actual inconsistency in Arithmetic, right?

No, because we are talking about the metalanguage here, not Arithmetic itself.

What we talk about, the definition of truth and provability, their relationship, is just like the axioms, so that they are part of the metalanguage or the metalogic involved, not the logic or the language itself, if that makes sense.

We use CL to deal with the own Arithmetic and we cannot get confused and extend that to the metalanguage or metalogic or what should be axiom.

If we say that whatever is true in Arithmetic has a proof, and whatever has a proof is true, that may, in our minds, directly connect to an iff, but, that is part of the Purely Human Language, not of the mathematical one, if that makes sense. In this case, we cannot and we should not dare proving that with CL, since this is beyond CL: Only Mathematics itself has to obey CL, not the axioms or the foundations of it.

That would be the same as saying summing is changing numbers into matches, then seeing the corresponding number in the scale to the total amount of matches we have, and then transferring it to CL: Do it!

There is a confusion here, which is precisely talking about what is the foundations of a building as if those were the own building.

The building works in a way, the foundations work in another way, and those ways may be independent. It is not because we have a lift inside of the building that we will have one inside of the ground, where it is coming from, right?

It is not because we have to go deep, whatever amount of meters, not to have the building falling to the ground when we use it, that we will have to have that same amount of meters to hold the floor that goes inside of the building itself, right?

What is meant here is that different rules apply to foundations and building.

In any hypothesis, our foundation would say that $P \vdash(\varphi \leftrightarrow \operatorname{Prov}(/ \varphi)))$, not that $P \vdash(\varphi \leftrightarrow \neg \operatorname{Prov}(/ \varphi))$.

We must observe that this definition is valid for the entire whole of Mathematics, not only for Arithmetic, so that if there were a flaw there, the flaw would be present in any piece of Mathematics as well. 
$P \vdash(\varphi \leftrightarrow \neg \operatorname{Prov}(\lceil\varphi))$ is not a valid sentence in CL because it voids the rules for implications, as pointed out before. For us to accept a sentence inside of a certain logical system, we must follow its rules, and this sentence clearly violates those.

We can put symbols together and write whatever we want in CL, so say $2+5=7 \leftrightarrow \neg \operatorname{Prov}([2+5=7])$. We know that this is not true, for there is a proof that $2+5=7$ instead. We here have a clearly false iff. Because this is one instance of the previous iff, we now know that $P$ could never support our tautology, right? That is how we prove that we cannot really write such a thing in CL, and therefore that Gödel DID NOT have a counter-example or something that could allow him to assert the inconsistency of Peano's Axioms. In this case, we do not yet have an incompleteness theorem for Arithmetic. All we know tells us precisely the opposite: Arithmetic is complete.

We further observe that what Stanford claims to be a proof of inconsistency of Arithmetic written by Gödel looks like a standard absurdity-based proof (if we suppose that it is true, we get inconsistency. Therefore, we cannot have that being true. The implication is then that there is no logical expression that is true, valid, and not provable in Arithmetic).

Meyer [14] states that Gödel's proof needed fixing, but the conclusions would still be the same, if we understood him well. See:

(Page 39)

For $\boldsymbol{x}=\boldsymbol{q}$ either:

(A.3.15) Bew $\{S b[q, 17, Z(q)]\} \Rightarrow B e w\{S b[q, 17, Z(q)]\}$, or

(A.3.16) $\neg \operatorname{Bew}\{S b[q, 17, Z(q)]\}$

It follows that (A.3.15) cannot apply, since that is a straightforward contradiction. Therefore it must be the case that (A.3.16) applies. This means that the relation $\neg \mathbf{B e w}\{\mathbf{S b}[\mathbf{q}, \mathbf{1 7}, \mathbf{Z}(\mathbf{q})]\}$ holds. Since that is the case, then there cannot be a formal proof of the formal formula that corresponds to $\mathbf{S b}[\mathbf{q}, \mathbf{1 7}, \mathbf{Z}(\mathbf{q})]$.

Now the formal formula that corresponds to $\mathbf{S b}[q, \mathbf{1 7}, \mathbf{Z}(\boldsymbol{q})]$ is the formal formula that corresponds to the relation $\boldsymbol{R}(\boldsymbol{q})$. But the relation $\mathbf{R}(\boldsymbol{q})$ is actually the relation $\neg \mathbf{B e w}\{\mathbf{S b}[\boldsymbol{q}, \mathbf{1 7}, \mathbf{Z}(\mathbf{q})]\}$, which does hold true, as given by (A.3.16).

Therefore the relation $\mathbf{R}(\boldsymbol{q})$ holds true, and the formal formula that corresponds to $\mathbf{R}(\boldsymbol{q})$ must also be true, but there cannot be a formal proof of the formal formula that corresponds to $\boldsymbol{R}(\boldsymbol{q})$.

This completes the proof. It will be observed that the above proof requires no additional assumptions that are not of the same nature as those used in Gödel's original proof.

(Page 12)

Bew $\{S b[Q 1, U, Z(X)]\}$ corresponds to Provable[Subst(F1, fv, X)]

(6.2.13) for the 'number-theoretic relation' $\mathbf{R} 1$, there is a corresponding formal formula $\mathbf{F} \mathbf{1}$, and Subst(R1, fv, $X)$ corresponds to Subst $(F 1, f v, X)$,

(6.2.14) for the formal formula $\mathbf{F 1}$, there is a corresponding Gödel number $\mathbf{Q 1}$, and $\mathbf{S u b s t}(\mathbf{F 1}, f v, X)$ corresponds to $\mathbf{S b}[\mathbf{Q} 1, \boldsymbol{U}, \mathbf{Z}(X)]$,

(6.2.15) Provable[Subst(F1, fv, X)] [7] corresponds to Provable[Subst(R1, fv, X)]

(Page 19)

$17=\Psi\left(v^{F}\right)$

(Page 6)

the function $\boldsymbol{\Phi ( X )}$ references by the function $\boldsymbol{\Psi}(\boldsymbol{t})$ every symbol of the symbol sequence $\boldsymbol{X}$.

(Page 5)

$\Psi(X)$ A function that gives a specific natural number of the metalanguage for every symbol of the formal system

$T^{A}$ A mapping function

$\boldsymbol{x}$ Proof ${ }^{F} \boldsymbol{y}$ The symbol combination $\boldsymbol{x}$ is a proof in the system $\mathrm{F}$ of the symbol combination $\boldsymbol{y}$

$\boldsymbol{F r}(\boldsymbol{x}, \boldsymbol{y}) \boldsymbol{x}$ is a free variable in the symbol sequence $\boldsymbol{y}$

This is very different from what we see in [1], and also very different from what we see in [15].

Meyer [14] seems to be talking about something simply unprovable but true.

There is a number for such a formula, it is true therefore, but there is no proof for it.

Such a thing could be, for instance, that parallels do not meet on infinity. Of course, the reason that we don't have a proof for this one is probably because it is missing to define what a proof is when we have infinity in terms of length and geometric objects to deal with.

If we say that proving that is considering a slice of the event and then reasoning that every other slice will be a 
copy, what then gives us, in an absolute manner, that they don't meet on infinity, then we have a proof, is it not?

It is still not something that we can prove and people usually say that they actually meet on infinity, this probably considering Biology and usual eyesight capabilities (our eyes will make us think that they meet on infinity, so that it all depends on whether we can go to the end when we tell if they meet or not or we are stuck with remaining at some static point of those, right?).

Axioms are not provable and should be considered part of the logical system. We can obviously use Gödel's coding system to translate the definition of power [16]: We can say that $0^{n}=0 \times 0 \times 0 \ldots \times 0$ ( $n$ times) or something like that, then assign a function to refer to each symbol in this string, and so on. It is true, but it is unprovable because it is an axiom.

If we split axioms from derivations of those, however, we should end up with complete as a result. It does not suffice saying that something is unprovable. We have to say what that something is to prove incompleteness, for all axioms are part of the system and are, by default, unprovable items.

Once more, these axioms are part of what we could call metalogic, since they are definitions of higher order, let's say. It is just like God: No proof, we simply accept.

We obviously do not have anything in Arithmetic that is unprovable if all things considered are part of the derivations, since, by default, derivation is something that came from something else. In our case, it would have come from the axioms.

If we think that the fact that the axioms are unprovable makes our system incomplete, then all the systems ever created will be incomplete, not only Arithmetic.

Astonishingly, Meyer [14] does not bring a definition for incompleteness.

Not to be believed, this is part of the confusion: The most important logicians in 2016, just like Graham Priest, define completeness as going upwards in the deductive chain and soundness as going downwards, basically.

In this case, every true and valid statement has a root on some axiom or set of those implies that the system is complete.

Recall now that, for Gödel, a logical expression is a well-formed first order formula without identity. A valid expression is true in every interpretation and a satisfiable expression is true in some interpretation. An expression is refutable (false) if its negation is provable.

When we say that $P(x)$ is not true, that is, that a certain arithmetic proposition is not true, let's say, then we should be basing ourselves in some axiom or set of axioms to say so. That should always be possible.

We see a bit of a problem between the concepts of validity here if Kennedy [1] is correct:

$A->B$ would not be true in every interpretation, since if $v(A)=1$ and $v(B)=0$, then $v(A->B)=0$, as for what Priest and his fellows think nowadays. Priest checks on the deduction of this formula to tell if a chosen logical system is complete or not.

A valid logical expression, according to Kennedy’s reading of Gödel's work [1], would be a tautology. We should then check only on the deduction of those to determine completeness if she is right.

Notwithstanding, every tautology would also be a satisfiable expression, since those are obviously weaker in terms of truth. In this case, if we check all satisfiable expressions, which is what Priest and his fellows do, we would also satisfy Gödel's completeness requirements.

The problem with the proof Meyer [14] attributes to Gödel is that he starts with $v(P(x))=1$ but there is no proof, or sequence of inferences from a basic axiom, for $P(x)$.

Who is $P(x)$ ?

We don't know.

It is 17 .

If we don't know who 17 is, how can we tell if it is true or not?

Then they will say: Assume that there is a $P(x)$ that is not provable.

We cannot assume what we want to prove, basically.

We are defining truth as being the same as provable unless we have an axiom, so that this is absurd.

If it is not provable, then we cannot attribute a valuation 1 to it, quite trivially. Then Gödel seems to be saying that because we can code this assertion by means of symbols, it is true, like it is in our list.

Well, $4+2=1$ would also be in our list.

That is ALSO not true.

Yet, it is allowed in the system and would deserve a Gödel's number.

It would also be unprovable because it is not true. 
We could then have $17=(4+2=1)$, is it not?

17 is unprovable for sure, will have a number in that list, and that means nothing.

To test completeness, according to Priest, we have to choose true sentences, not only valid, allowed by the system, but true.

$4+2=1$ is not true, so that $v(4+2=1)=0$. It is not provable, so that $\neg \boldsymbol{B e w}\{\boldsymbol{S} b[\boldsymbol{q}, \mathbf{1 7}, \mathbf{Z}(\boldsymbol{q})]\}$ and $\neg \operatorname{Bew}\{\operatorname{Sb}[Q 1, U, Z(X)]\}$ corresponds to Not Provable $[S u b s t(F 1, f v, X)](6.2 .13)$ for the number-theoretic relationR1, there is no corresponding formal formula $F 1$, such that $\operatorname{Subst}(4+2=1, f v, X)$ orresponds to $\operatorname{Subst}(F 1$, $f v, X)$, let's say.

So?

$4+2=1$ is number 17 in the list!

Of course, of course, we will say.

Now, completeness and consistency are slightly different concepts: Whilst completeness has to do with every true proposition being deductible from the basic axioms of the system, consistency has to do with it not being possible that we deduce both the truth and the falsity of something in the case of Classical Logic.

Read the following extract (Figure 1), which came from the second page of [17]:

\section{Hilbert, Gödel, and the Consistency of Arithmetic}

In 1900, David Hilbert presented a list of 23 unsolved mathematical problems to the Paris conference of the International Congress of Mathematicians. The second problem on this list was to prove the consistency of the arithmetical axioms, i.e. to show that from the axioms no pair of theorems |- A, and |- not-A, were both derivable. In Hilbert's words:

But above all I wish to designate the following as the most important among the numerous questions which can be asked with regard to the axioms: To prove that they are not contradictory, that is, that a definite number of logical steps based upon them can never lead to contradictory results.

From 1900 to 2011 Hilbert's second problem has remained unresolved, and his program of proving arithmetical consistency unachieved. The conventional wisdom regarding it is that in 1931 Kurt Gödel showed that it was unachievable, i.e. that even if arithmetic were consistent, this fact could never be proved. Gödel's proof falls into the same category as another well-known impossibility proof: the impossibility of deriving Euclid's parallel postulate from the other axioms of geometry.

Figure 1. No possible proof.

It seems that McCall [17] is actually saying that proving that Arithmetic is consistent is not something that we can do and that Gödel would then have exposed a possible flaw, but not necessarily a flaw.

We observe that what was being said, before this extract, was not that Gödel said that it was possible that Arithmetic were inconsistent, just that if it is consistent, then maybe we cannot prove it. In McCall [17], we see, on page 3 (Figure 2).

Does this mean that Hilbert's Program, calling for an axiomatic formalization of mathematics together with a proof that the resulting system engenders no contradictions, is doomed to failure? Not necessarily. In essay 1 below is found a different kind of consistency proof of arithmetic, a proof based not on deriving theorems from axioms but on a semantic model that includes a non-linguistic domain of objects. This proof satisfies Hilbert's requirement of finitism, namely that the overall argument, and each of the sub-proofs on which it is based, should consist of a finite number of steps. A key requisite is that every model in the semantics for PA must be based on a finite domain. The conclusion is, that despite Gödel's incompleteness theorems of 1931, the consistency of arithmetic is provable. The proof requires that PA's mathematical axioms, symbols and syntax be supplemented by a non-linguistic, semantic domain of objects. 
Our work started in the year of 2008 in what regards completeness of Arithmetic and its proof, and we had it exposed online back then. We suffer really serious crime, so that other people could eventually end up doing something similar or even better during that time, is it not? McCall [17] seems to be referring to this sort of thing.

We totally believe that the consistency of Arithmetic is both provable and evident. We observe, however, once more, that completeness and consistency are two very different concepts. We worry about completeness, but McCall seems to be worried about consistency.

To prove incompleteness, we just need to exhibit one allowed and true statement that is not deductible from the axioms of the system.

To prove completeness, we would probably adopt the concept of Priest and then depart from every possible formula, created using the allowed connectors and symbols, to then go upwards/backwards aiming at the elementary roots. This process should allow us to put together a deduction for each one of those (it suffices taking note of the steps we took and then go backwards).

To prove consistency, since Arithmetic is founded on Classical Logic, we must prove that, for all allowed propositions, we get one, and at most one of the options, validated: It is true, and it is false.

\section{Conclusions}

We conclude that Gödel DID NOT have any acceptable piece of evidence in the direction of Arithmetic being incomplete. Human kind ALSO never found such a piece of evidence, so that Arithmetic must be complete.

We will probably attempt to prove such a thing in the future.

We also conclude that the current scientific literature might bring a bit of confusion in what regards the terms complete and consistent: One concept differs, in a fundamental manner, from the other.

There seems to be a generalized agreement ([15], [17], and [18]) in what regards the work of Gödel: That maybe he had a sentence that was unprovable and true, but we cannot really tell what this sentence is from reading his writings.

The sentence that Stanford [1] points at, This sentence is/I am not provable, is not actually acceptable because the sigmatoid sentence AND the sigmatoid $I$, as well as other sigmatoids, such as $A M$, do not belong to the Classical Logic lingo [11]. If we replace it with symbols, as Meyer [14], for instance, has done, then the problem becomes another: The argument is not really true if it involves the relationship between provable and true.

Meyer [14] claims to have used exactly the same symbols and writing used by Gödel. If that is true, then Gödel DID NOT have an argument that could convince us that Arithmetic is incomplete.

People who have dealt with Gödel's theory in the past seem to have confused metalanguage/metalogic with lingo and logical system, so that they applied the Classical Logic rules to what is outside of Arithmetic, what actually defines it, but those rules are valid exclusively inside of Arithmetic, not where we define it. The own Classical Logic (not its application, its definition instead) would be to that higher level, the level to which the metalogic belongs. We cannot make use of it at that stage, if that is clear enough.

Besides, when one attempts to do that, the Classical Logic rules for implications are violated, what then changes the only statement they had as an example of proposition that would be true but not provable into something disallowed or invalid, and therefore into example of nothing that matters.

Perhaps the worst difficulty people had so far was providing a simple example, practical: An example that could prove to everyone that such a possible proposition would not really be possible. We here came up with such an example: $2+5=7 \leftrightarrow \neg$ Prov $(\lceil 2+5=71)$.

If we understood the work of Meyer [14] well, we would also have a counter-example to his claim, which would actually be an instance of the main proposition, but this main proposition is presented as the greatest piece of evidence that Arithmetic is incomplete:

$4+2=1$ is not true, so that $v(4+2=1)=0$. It is not provable, so that $\neg \boldsymbol{B e w}\{\boldsymbol{S b}[\boldsymbol{q}, \mathbf{1 7}, \mathbf{Z}(\boldsymbol{q})]\}$ and $\neg \operatorname{Bew}\{\operatorname{Sb}[Q 1, U, Z(X)]\}$ corresponds to NotProvable[Subst(F1, fv, X)](6.2.13) for the "number-theoretic relation” $\mathbf{R} \mathbf{1}$, there is no corresponding formal formula $\mathbf{F 1}$, such that Subst $(4+2=1, f v, X)$ corresponds to $\operatorname{Subst}(F 1, f v, X)$.

\section{Acknowledgements}

We would like to thank Academia.edu and its platform as well as Prof. John Corcoran for having inspired us in what regards this paper. We also would like to thank the so dedicated editorial board of Advances for their continued support. 


\section{References}

[1] Kennedy, J. (2015) Kurt Gödel. http://plato.stanford.edu/entries/goedel/\#BioSke

[2] Gödel, K. (1929) “I”. University of Vienna, Vienna.

[3] Hilbert, D. and Ackermann, W. (1928) Grundzüge der theoretischen Logik. Springer-Verlag, Berlin.

[4] van Heijenoort, J., Ed. (1967) From Frege to Gödel: A Sourcebook in Mathematical Logic, 1879-1931. Harvard University Press, Cambridge.

[5] Zach, R. (1999) Completeness before Post: Bernays, Hilbert, and the Development of Propositional Logic. Bulletin of Symbolic Logic, 5, 331-366. http://dx.doi.org/10.2307/421184

[6] Post, E.L. (1921). Introduction to a General Theory of Elementary Propositions. American Journal of Mathematics, 43, 163-185. http://dx.doi.org/10.2307/2370324

[7] Bernays, P. (1926) Axiomatische Untersuchung des Aussagen-Kalkuls der "Principia Mathematica.” Mathematisches Zeitschrift, 25, 305-320. http://dx.doi.org/10.1007/BF01283841

[8] Godel, K. (1930) Die Vollständigkeit der Axiome des logischen Functionenkalküls. Monatshefte Für Mathematik Und Physik, 37, 349-360. http://dx.doi.org/10.1007/BF01696781

[9] Mal'cev, A.I. (1971) The Metamathematics of Algebraic Systems. In: Wells III, B.F., Ed., North-Holland Publishing Co., Amsterdam.

[10] Pinheiro, I.M.R. (2012) Concerning the Solution to the Liar Paradox. E-Logos, 21, 15. http://www.academia.edu/9787988/Solution_to_the_Liar_Paradox

[11] Pinheiro, M.R. (2015) Words for Science. Indian Journal of Applied Research, 5, 19-22. https://www.worldwidejournals.com/ijar/articles.php?val=NjQ0MQ==\&b1=853\&k=214

[12] Weisstein, E.W. (2016) Peano’s Axioms. http://mathworld.wolfram.com/PeanosAxioms.html

[13] Schechter, E. (2005) Classical \& Nonclassical Logics an Introduction to the Mathematics of Propositions. http://www.math.vanderbilt.edu/ schectex/logics/

[14] Meyer, J.R. (2009) The Fundamental Flaw in Gödel’s Proof of His Incompleteness Theorem “on Formally Undecidable Propositions of Principia Mathematica and Related Systems". https://www.academia.edu/4706759/The Fundamental Flaw in G\%C3\%B6del s Proof of the Incompleteness Theorem

[15] Pinheiro, M.R. (2008) On the Consistency and the Completeness of Arithmetic. Research Report, Washington Public Library, Washington, 32.

[16] MathsIsFun.com (2014) Definition of Power. https://www.mathsisfun.com/definitions/power.html

[17] McCall, S. (2014) Oxford University Pr. (The Consistency of Arithmetic: And Other Essays, Ed.). Oxford University Press. https://books.google.com.au/books?id=7d9_AwAAQBAJ\&pg=PR7\&lpg=PR7\&dq=mccall+2008+the+consistency+of + arithmetic\&source=bl\&ots=Nk4rx6vGc6\&sig=FQojeCga4E50uwRi5i3yU4lY2PM\&hl=en\&sa=X\&ved=0ahUKEwjn ov2gioPNAhWhg6YKHcakAVsQ6AEIGzAA\#v=onepage\&q\&f=false

[18] Pinheiro, M.R. (2016) Gödel and the Incompleteness of Arithmetic: He Had a Dream. http://itshouldallbeaboutlogic.blogspot.com.au/2016/05/godel-and-incompleteness-of-arithmetic.html

\section{Submit or recommend next manuscript to SCIRP and we will provide best service for you:}

Accepting pre-submission inquiries through Email, Facebook, Linkedin, Twitter, etc A wide selection of journals (inclusive of 9 subjects, more than 200 journals)

Providing a 24-hour high-quality service User-friendly online submission system

Fair and swift peer-review system

Efficient typesetting and proofreading procedure

Display of the result of downloads and visits, as well as the number of cited articles

Maximum dissemination of your research work

Submit your manuscript at: http://papersubmission.scirp.org/ 